\title{
Correlates of unprotected sexual intercourse among women who inject drugs or who have sexual partners who inject drugs in St Petersburg, Russia
}

\author{
Nadia Abdala, ${ }^{1}$ Nathan B Hansen, ${ }^{2}$ Olga $V$ Toussova, ${ }^{3}$ \\ Tatiana V Krasnoselskikh, ${ }^{4}$ Sergei Verevochkin, ${ }^{5}$ Andrei P Kozlov, ${ }^{6}$ \\ Robert Heimer ${ }^{7}$
}

${ }^{1}$ Research Scientist, Yale School of Public Health, New Haven, CT, USA

${ }^{2}$ Associate Professor, Department of Psychiatry, Yale University School of Medicine,

New Haven, CT, USA

${ }^{3}$ Senior Research Scientist,

The Biomedical Center,

St Petersburg, Russian

Federation

${ }^{4}$ Senior Investigator, The

Biomedical Center,

St Petersburg, Russian

Federation

${ }^{5}$ Head of the Clinical and

Diagnostic Laboratory,

The Biomedical Center

St Petersburg, Russian

Federation

${ }^{6}$ Director, The Biomedical

Center, St Petersburg, Russian Federation

${ }^{7}$ Professor, Yale School of Public Health, New Haven, CT, USA

\section{Correspondence to \\ Dr Nadia Abdala, \\ Yale School of Public Health, 60 College Street, 507 LEPH, New Haven, CT 06511, USA; nadia.abdala@yale.edu}

Received 16 December 2011 Revised 18 May 2012 Accepted 14 August 2012 Published Online First 2 February 2013

To cite: Abdala N, Hansen NB, Toussova OV et al. J Fam Plann Reprod Health Care 2013:39: 179-185.

\begin{abstract}
Objectives To assess risk for unintended pregnancy, this study describes the correlates of unprotected sexual intercourse (UPSI) among women who inject illicit drugs or who have sexual partners who inject drugs in St Petersburg, Russia.
\end{abstract}

Methods Data from a cross-sectional survey and biological test results collected between 2005 and 2008 from 202 Russian women (143 drug injectors and 59 non-drug injectors) were analysed. Multivariate regression was used to investigate the correlates of UPSI occurring at the women's last sexual act. Independent variables included socio-demographics, age at sexual debut, first sexual encounter perceived as involuntary, number of pregnancies and number of children for which the participant is the primary caretaker, heavy sporadic drinking (i.e. consuming more than five drinks in 2 hours at least twice a month), at-risk drinking per the Alcohol Use Disorder Identification Test (AUDIT-C) score, and sexually transmitted infections (HIV-1, syphilis serology, Chlamydia trachomatis and Neisseria gonorrheae).

Results Sixty-seven percent of women reported UPSI at last intercourse. UPSI was independently associated with heavy sporadic drinking [odds ratio (OR) 2.8, 95\% $\mathrm{Cl} 1.2-6.6$ ] and having been pregnant (OR 2.25, 95\% Cl 1.1-4.6).

Conclusions Despite the high risk for HIV acquisition or transmission and unintended pregnancy, condom use among the study population is low. Programmes to investigate and improve contraceptive use, including condom use, among this vulnerable group of women are needed. Such programmes may require

\section{KEY MESSAGE POINTS}

- Despite the high risk for HIV transmission and unintended pregnancy, the prevalence of condom use was low among participants, independent of women's HIV status.

- Programmes to investigate and improve contraceptive use, including condoms, among this vulnerable group of women are needed and might benefit from addressing alcohol misuse.

- Studies that investigate reproductive health issues, including early sexual behaviour and substance use patterns, are needed among Russian women at high risk for HIV.

identifying and targeting female reproductive health concerns and problem drinking,

particularly heavy sporadic drinking, rather than conventional measures of alcohol misuse.

\section{INTRODUCTION}

Russia has one of the highest HIV incidence rates in Eastern Europe and Asia. ${ }^{1}$ Although the HIV epidemic in Russia is concentrated among injection drug users (IDUs), data from the Russian Ministry of Health show an increase in the rate of sexually acquired HIV infections among women ${ }^{2}{ }^{3}$ and a growing number of children born to HIV-infected mothers. ${ }^{4}$ The HIV infection levels measured among 
pregnant women rose from less than $0.01 \%$ in 1998 to $0.11 \%$ in $2003 .^{5}$ For these reasons, studies that investigate reproductive health issues among Russian women who inject drugs or who are sexual partners of men who inject drugs may provide useful HIV prevention information.

Because induced abortion was the most common means of preventing births during the Soviet period, ${ }^{6}$ Russia has one of the highest abortion rates in the world today. ${ }^{7}$ Although abortions can be recommended and performed for medical reasons, most abortions in Russia occur due to unintended pregnancy. ${ }^{7}$ Among the general female population in large urban areas, the self-reported prevalence of abortion approximates $50 \% .{ }^{8}$ Among more vulnerable populations, such as female IDUs, the abortion prevalence may reach $67 \% .^{9}$ Efforts to decrease unwanted pregnancies between 1991 and $1997^{10} 11$ apparently led to a reduction in the number of abortions and better attitudes toward contraceptive methods in Russia. ${ }^{12} 13$ However, results from a longitudinal monitoring survey between 1994 and 2003 revealed that effective contraceptive use had not increased among sexually active Russian women. ${ }^{14}$

The refusal to use contraceptives and the resulting risk for unintended pregnancy has several negative consequences for women. Unintended pregnancy is associated with higher rates of negative maternal behaviours [e.g. failure to resolve sexually transmitted infections (STIs) prior to pregnancy, substance use during pregnancy, and a lower number of prenatal care visits], and a greater risk for the abandonment of infants and abortion. ${ }^{4}{ }^{15-18}$ In Russia, where rates of alcohol consumption are among the highest in the world, ${ }^{19}$ alcohol misuse may compound women's reproductive health-related problems. Alcohol misuse is more likely to occur during an unintended pregnancy, as are alcohol-related negative birth outcomes including birth defects ${ }^{20} 21$ and a range of developmental disabilities and fetal alcohol spectrum disorders. $^{22}$ Among non-pregnant women, misuse of alcohol has been associated with unplanned intercourse, ${ }^{23}$ unprotected sexual intercourse (UPSI), lack of contraceptive use, ${ }^{18} 24$ and with being diagnosed with an STI, ${ }^{23}$ and may compound women's risks for unintended pregnancies, abortions, reproductive health problems and HIV transmission. Thus, the investigation of the association of alcohol use to women's reproductive health issues may provide useful public health information.

Other factors that may contribute to women's patterns of contraceptive use, such as early sexual behaviour, have not been extensively investigated in Russia. For example, in a US study, ${ }^{25}$ adolescents who initiated sex at a younger age were more likely to be frequent polysubstance users and to not have used condoms during their last episode of intercourse. Other studies have linked childhood sexual trauma to adult sexual risk-taking and adult substance abuse. ${ }^{26-28}$ Substance use such as illicit drug injection, methamphetamine use and problem drinking may also contribute to unsafe sex. ${ }^{29-31}$ Therefore studies of women's reproductive health risks may benefit from controlling for patterns of substance use and for early sexual behaviours and trauma. Such studies may provide helpful information for the development of interventions to increase contraceptive use, including condom use among vulnerable women.

Despite these findings, no study has yet investigated the relationship between lack of contraceptive use and the factors related to the reproductive health and early sexual behaviours of women who are at elevated risk for HIV and unintended pregnancy in Russia. We conducted an initial study to investigate the correlates of unsafe sex, including early sexual behaviour, reproductive health characteristics and patterns of alcohol use among women who inject drugs or who have sexual partners who inject drugs in St Petersburg, Russia.

\section{METHODS}

\section{Study population and recruitment into the SATHCAP study}

This analysis uses data from a study conducted in St Petersburg, Russia, which was part of a large multisite project, the Sexual Acquisition and Transmission of HIV-Cooperative Agreement Program (SATHCAP). ${ }^{32}$ The goal of SATHCAP was to recruit individuals who inject drugs, and their sexual partners, and collect data on the factors that may influence the transmission of HIV from high-prevalence core populations (such as IDUs) to the greater general populations of which they are part.

The SATHCAP study protocol was approved by the Institutional Review Boards of Yale University, the Biomedical Center in St Petersburg, and RAND Corporation. Participants were recruited between November 2005 and December 2008 using a modified form of respondent-driven sampling (RDS). ${ }^{32} 33$ RDS is a chain-referral sampling methodology that uses structured incentives and coupon disbursement for peer referrals. Conventional RDS methods were modified $^{32}$ in this study to recruit IDUs and their sex partners. Enrolled IDUs were given coupons to distribute to their peers and to their sex partners. After receiving coupons, potential participants contacted the study site to schedule an appointment for eligibility screening and enrolment. Newly enrolled IDUs were in turn offered coupons to recruit their sexual partners and additional injection drug-using peers. Participation was anonymous. No locator information was collected but a number of bio-measures including forearm length and wrist circumference were collected to prevent repeat participation (duplicates). ${ }^{34} 35$

After providing written informed consent, participants completed structured interviews and were given pre-test counselling before biological specimens were 
collected for the testing of STIs, including HIV. Participants received incentives equivalent to US $\$ 10$, which included mobile phone cards or personal care items; all received subway tokens, condoms and HIV prevention information. Participants were instructed when to return to the site to receive their laboratory test results and post-test counselling. Participants were referred for other medical services as needed.

\section{Interview data collection}

Interviews lasting 90-120 minutes were conducted using computer-assisted survey interviewing technology on laptop computers. Information was collected on socio-demographics, health status and HIV-associated sexual and injection risk behaviours. Socio-demographic data included age, sex, education and employment. Participants were asked if they had ever been tested for HIV. If a participant had been previously tested for HIV, he or she was asked for the result of the HIV test from a list of five options: refuse to answer, did not know, did not get results, HIV-negative and HIV-positive.

Participants were asked if they injected illicit drugs in the last 6 months. They were asked the three first questions of the Alcohol Use Disorder Identification Test (AUDIT-C): the number of days in the last month during which alcohol was consumed, average number of drinks at each drinking event, and number of days in the last month during which they got drunk or consumed five or more drinks within 2 hours. Heavy sporadic drinking was defined as being drunk or having five or more drinks within 2 hours at least twice a month. At-risk drinking corresponded to a score greater than two on the AUDIT-C questions as determined in previous studies among women. ${ }^{36} 37$

Information on sexual behaviours included the number of male and/or female sex partners in the 6 months prior to the interview. Participants were asked whether they had unprotected vaginal or anal sexual intercourse (UPSI) at the last sexual encounter with up to five recent sexual partners within the prior 6 months. For each reported partnership, the participants were asked whether they knew the HIV sero-status of their sexual partners. Self-reported sexual and reproductive characteristics included age at first sexual intercourse, whether participants perceived their first sex to have been involuntary, current pregnancy status, the number of children for whom the participant is the primary caretaker, and number of previous pregnancies.

\section{STI detection assays}

Blood and urine specimens were obtained from all participants. Sera were tested for both HIV type-1 (HIV-1) and syphilis. HIV-1 testing involved two HIV-1 antibodies enzyme immunological assays (EIAs) (Vironostika HIV-1, Uni-Form-II plus-0; Biomerieux, The Netherlands and Genscreeen HIV-1/2; BioRad, France). Positive HIV-1 EIA tests were confirmed by
Western Blot (WB) (HIV-1 WB Type-1; Biorad, France). Specimens that were positive by WB were considered HIV-positive. Serum samples were screened for syphilis with rapid plasma reagin test (RPR) (Macro-Vue RPR-Card Tests; Becton Dickinson, USA) and Treponema pallidum particle agglutination assay (TPPA) (Serodia-TPPA; Fujirebio, Japan). Specimens were seropositive for syphilis if TPPA alone or if both TPPA and RPR tests were reactive. Urine specimens were tested for nucleic acid from Chlamydia trachomatis and Neisseriae gonorrheae using polymerase chain reaction (PCR) (Amplicor CT/ NG; Roche, USA).

\section{Data analysis}

Because equilibrium in RDS was not satisfactorily achieved, the presented results do not include standard RDS weighing algorithms. Standard descriptive statistics were used to describe the sample. Bivariate associations between UPSI and independent variables, such as socio-demographics, substance use, sexual and reproductive characteristics and HIV/STIs, were examined. Variables associated with UPSI at $p<0.20$ were entered into multivariate logistic regression models. Backward stepwise elimination was used to produce adjusted ORs for those variables independently associated with UPSI at $p$ values $\leq 0.05$. All data were analysed using SPSS Version 17 (IBM Corporation, USA).

\section{RESULTS}

\section{General characteristics and substance use patterns} of subjects

In all, 1023 IDUs, men who had sex with men and their sexual partners were recruited into the completed SATHCAP study in St Petersburg. After excluding all males (772), two transgendered individuals (2), those who reported zero male sex partners (71), those who did not report their number of sex partners in the prior 6 months (34), and those who did not answer questions about their sexual partnerships (78), 202 participants were included in the analysis (note that some participants belonged to more than one of these categories). This included 143 (71\%) women who reported injection drug use in the prior 6 months and $59(29 \%)$ women who did not. The ages of all participants included in this analysis were in the range 18-46 years (Table 1), with a median of 26 years [interquartile range $(\mathrm{IQR})=22-31$ ]. Most participants had completed secondary education and were employed. Furthermore, $25 \%$ of the women engaged in heavy sporadic drinking and $65 \%$ received an at-risk drinking score.

\section{Sexual and reproductive characteristics and STI test results}

The median age for the first sexual act was 16 (IQR $=14-17$, min 11-max 23), 13\% perceived their first sexual encounter to have been involuntary, and 


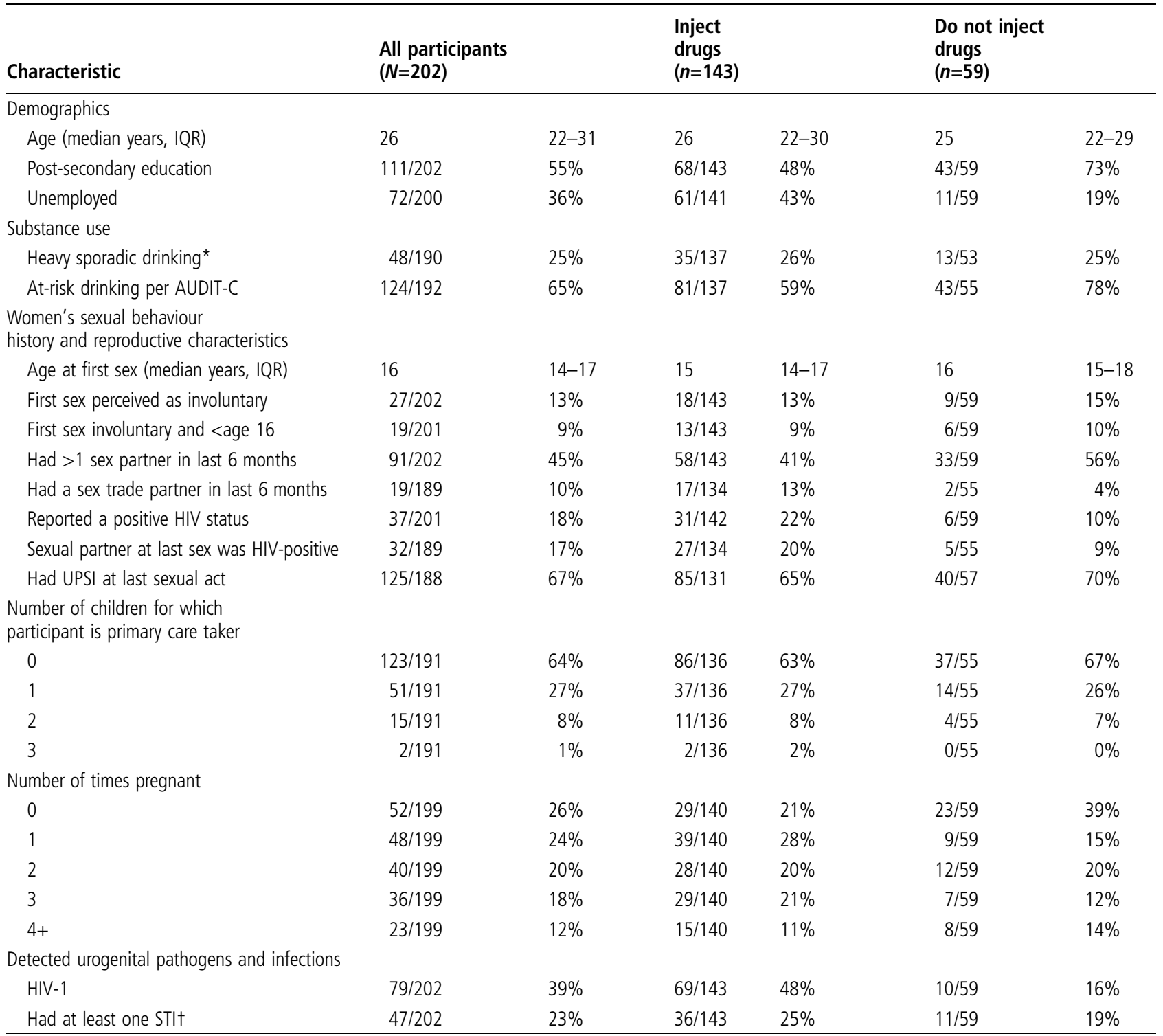

${ }^{*}$ Drunk or had five or more drinks in 2 hours at least twice a month.

tChlamydia trachomatis, Neisseria gonorrhoeae or positive syphilis serology.

IQR, interquartile range; SATHCAP, Sexual Acquisition and Transmission of HIV-Cooperative Agreement Program; STI, sexually transmitted infection;

UPSI, unprotected sexual intercourse.

9\% reported that their sexual debut was involuntary and occurred when they were younger than age 16 years (Table 1). Nearly half of all women had more than one sex partner in the last 6 months and $10 \%$ had a sex trade partner (a partner with whom they exchanged money, goods or drugs for sex). Nearly two-thirds reported having had UPSI at their last sexual intercourse, $18 \%$ self-reported having a positive HIV status (though 39\% were found to be positive at HIV testing) and $17 \%$ reported that the sexual partner with whom they last had sex was HIV-positive. Overall, $11 \%$ and $10 \%$ of participants tested positive for HIV, or reported being positive for HIV, respectively, and also reported having had a sexual partner who was HIV-positive.
While $64 \%$ of all participants had no children, 74\% reported having been pregnant. Further, while 1\% of participants reported at least three children, $30 \%$ had been pregnant three or more times. Laboratory test results revealed an HIV prevalence of 39\% (48\% among injectors and 16\% among non-injectors) and $23 \%$ tested positive for chlamydia, gonorrhoea or syphilis serology.

\section{Correlates of UPSI as marker for pregnancy risk}

In the bivariate analysis among all participants (Table 2), UPSI at the last sexual act was significantly associated with heavy sporadic drinking and with having been pregnant. In the multiple logistic regression model, heavy sporadic drinking (OR 2.8, 95\% CI 
Table 2 Correlates of unprotected sexual intercourse at last sexual act among female SATHCAP participants who inject drugs or who have sexual partners who inject drugs

\begin{tabular}{|c|c|c|}
\hline \multirow[b]{2}{*}{ Characteristic } & \multicolumn{2}{|c|}{ Among all women $(N=202)$} \\
\hline & uOR & $\mathrm{aOR}$ \\
\hline \multicolumn{3}{|l|}{ Demographics } \\
\hline Age $25+$ years & \multicolumn{2}{|l|}{$0.72(0.39-1.31)$} \\
\hline Post-secondary education & \multicolumn{2}{|l|}{$0.81(0.44-1.50)$} \\
\hline Unemployed & \multicolumn{2}{|l|}{$1.41(0.74-2.70)$} \\
\hline \multicolumn{3}{|l|}{ Substance use } \\
\hline $\begin{array}{l}\text { Injection drug use in last } \\
6 \text { months }\end{array}$ & \multicolumn{2}{|l|}{$0.78(0.40-1.54)$} \\
\hline Heavy sporadic drinking ${ }^{\dagger}$ & \multicolumn{2}{|c|}{$2.68(1.15-6.22)^{*} 2.80(1.18-6.60)^{*}$} \\
\hline At-risk drinking per AUDIT-C & \multicolumn{2}{|c|}{$1.45(0.77-2.76)$} \\
\hline \multicolumn{3}{|c|}{ Women's sexual behaviour and reproductive characteristics } \\
\hline First sex below age 16 years & \multicolumn{2}{|c|}{$1.47(0.80-2.71)$} \\
\hline $\begin{array}{l}\text { First sex perceived as } \\
\text { involuntary }\end{array}$ & \multicolumn{2}{|l|}{$2.34(0.84-6.54)$} \\
\hline $\begin{array}{l}\text { First sex below age } 16 \text { years } \\
\text { and involuntary }\end{array}$ & \multicolumn{2}{|l|}{$2.73(0.76-9.80)$} \\
\hline $\begin{array}{l}\text { Had more than one sex } \\
\text { partner in last } 6 \text { months }\end{array}$ & \multicolumn{2}{|l|}{$1.15(0.63-2.12)$} \\
\hline Reported a positive HIV status & \multicolumn{2}{|l|}{$1.27(0.57-2.86)$} \\
\hline $\begin{array}{l}\text { Had a sex trade partner in last } \\
6 \text { months }\end{array}$ & \multicolumn{2}{|l|}{$0.46(0.17-1.23)$} \\
\hline $\begin{array}{l}\text { Sexual partner at last sex was } \\
\text { HIV-positive }\end{array}$ & \multicolumn{2}{|l|}{$0.83(0.38-1.84)$} \\
\hline Has children & \multicolumn{2}{|l|}{$1.49(0.77-2.88)$} \\
\hline Has been pregnant & \multicolumn{2}{|c|}{$1.99(1.02-3.91)^{*} 2.25(1.11-4.58)^{*}$} \\
\hline \multicolumn{3}{|c|}{ Detected urogenital pathogens and infections } \\
\hline HIV-1 & \multicolumn{2}{|l|}{$1.33(0.71-2.49)$} \\
\hline Had at least one STI & \multicolumn{2}{|c|}{$1.15(0.56-2.37)$} \\
\hline \multicolumn{3}{|c|}{$\begin{array}{l}{ }^{*} p<0.05 . \\
\text { tDrunk or had five or more drinks in } 2 \text { hours at least twice a month. } \\
\neq \text { Chlamydia trachomatis, Neisseria gonorrhoeae or positive syphilis } \\
\text { serology. } \\
\text { aOR, adjusted odds ratio; SATHCAP, Sexual Acquisition and Transmission } \\
\text { of HIV-Cooperative Agreement Program; STI, sexually transmitted infection, } \\
\text { uOR, unadjusted odds ratio. }\end{array}$} \\
\hline
\end{tabular}

1.2-6.6) and having been pregnant (OR 2.2, 95\% CI 1.1-4.6) remained independently associated with UPSI at the last sexual act.

\section{DISCUSSION}

This is the first study to explore the relationships between women's risk for HIV and unintended pregnancy taking into consideration early sexual risk behaviours, parity and substance use patterns of women who are at high risk for HIV infection in St Petersburg, Russia. Nearly two-thirds of all participants reported UPSI at their last sexual intercourse. UPSI may be considered a strong indicator for pregnancy risk among this population. Although this study did not collect data on contraceptive behaviour other than condom use, a previous study of the contraceptive use patterns of 80 sexually active female IDUs from St Petersburg revealed that condoms were the only effective contraceptive method used by women. ${ }^{9}$ The findings suggest that women who inject drugs or who have sex with partners who inject drugs are at elevated risk for HIV acquisition and transmission and unintended pregnancy. Although the prevalence of risk behaviour was lower than the $88 \%$ prevalence of UPSI found among narcology patients in Russia, ${ }^{38}$ it is still high especially when considering that $39 \%$ of participants received a positive HIV test result in this study.

UPSI was independently associated with sporadic heavy drinking. The results contrast with a previous study among narcology patients in Russia that failed to detect an association between UPSI and binge drinking. ${ }^{38}$ However, our result is consistent with studies conducted outside Russia that show drinking to be associated with a greater chance of engaging in sexual risks, such as unplanned intercourse and UPSI. ${ }^{18} 3940$ These results indicate that programmes to increase condom use among women at high risk for HIV need to address problem drinking, particularly heavy sporadic drinking. The results showing no independent association between UPSI and risky drinking per AUDIT-C criteria suggest that interventions may need to identify and target populationspecific drinking patterns rather than conventional measures of problem drinking in order to achieve changes in sexual risk behaviours. Moreover, given that a previous study of female STI clinic patients in St Petersburg found an association between alcohol misuse and self-reported abortion, ${ }^{17}$ this finding suggests that studies to investigate the links between alcohol and contraceptive use patterns among this vulnerable group of women are needed.

UPSI at one's last sexual act was associated with having been pregnant. This is consistent with a previous study in Russia that found lower odds of contraceptive use among women who had given birth. ${ }^{8}$ The lack of condom use could be due to the use of other forms of contraceptive methods among the women who had been pregnant. However, a previous study among sexually active female drug injectors in St Petersburg showed that none of the participants used hormonal pills or intrauterine devices, ${ }^{9}$ which could also be the case in this study. The risk for unintended pregnancy can have marked negative consequences given that $18 \%$ of women reported being HIV-positive (and 39\% actually tested positive) and $10 \%(n=11 / 116)$ of those who tested negative for HIV reported that their partner at last sexual act was living with HIV. Thus, studies that investigate the contraceptive use patterns of this group of women are urgently needed.

The fact that a third of the participants reported three or more pregnancies, despite only $1 \%$ of women having three or more children, supports the possibility that the high number of pregnancies are a 
proxy for the number of induced abortions among participants. This is quite feasible given that abortion prevalence can be $50 \%$ or higher among Russian women. ${ }^{14}{ }^{41}$ However, there are other explanations for the discrepancy between the number of children and the number of pregnancies. Russia has high rates of abandonment of infants by HIV-positive women or women who did not intend to become pregnant; ${ }^{4} 42$ in addition, women who inject drugs may lose child custody ${ }^{43}$ and may be more prone to pregnancies that result in miscarriages, stillbirths or infant mortality. ${ }^{44}$ These possibilities are important public health concerns and need further scientific investigation.

Knowing one's HIV status to be positive, engaging in sex trade, or knowing that the sexual partner with whom one last had sex with had a positive HIV status were not associated with a greater likelihood of using condoms. While consistent and correct use of male condoms can provide effective protection against HIV and other STIs, they are associated with relatively high pregnancy rates compared to other contraceptive methods. ${ }^{45}$ In one study, the average failure rate for the male condom was estimated to be $15 \%{ }^{46}$ These results further confirm the great need for more efforts to increase condom use and dual contraceptive use among these women.

Approximately $13 \%$ of participants reported involuntary first sex and in approximately two-thirds of these cases this occurred before the age of 16 years. This number may approximate the $10 \%$ prevalence of forced sexual intercourse reported by female adolescents in the USA. ${ }^{47}$ However, additional questions about sexual coercion, abuse or assault were not asked in this study. Intimate partner violence and sexual violence in adulthood have been linked to risky sexual behaviour, and can restrict women's abilities to use contraception, including condoms. ${ }^{48}$ Additional studies will need to confirm the prevalence of sexual trauma and early sexual risk behaviours and their associated risk factors among this group of women.

\section{Limitations of study}

A primary limitation with this study is the small sample size, which restricted the analyses that could be conducted, as well as the power to identify small but significant relationships that may exist. There is a need for larger studies to confirm these results. Risk behaviours may have been under-reported because of social desirability or other types of bias. Due to the crosssectional nature of the data, causal relationships could not be established. Participants recruited into this study were drug injectors and non-injectors who reported at least one sexual partner who was an IDU; thus the results may not apply to individuals who never had a sexual partner who injected drugs. In addition, we did not ask participants about contraceptive use patterns or pregnancy desires, which limits the conclusions that can be drawn from our findings. Finally, the non- representative recruitment method may limit the generalisability of the results. However, the study provides a unique opportunity for the identification of reproductive health problems that may also place women at risk for HIV and unintended pregnancy.

\section{CONCLUSIONS}

Despite the high risk for HIV acquisition or transmission and unintended pregnancies, condom use among the study population is low. Heavy sporadic drinking and a history of pregnancies were associated with UPSI among this vulnerable group of women. Programmes to address alcohol misuse and women's reproductive health concerns, including the contraceptive decision-making of women who use injection drugs or have IDU sexual partners in St Petersburg, Russia, are needed.

Acknowledgements The authors want to thank the other SATHCAP sites for their support: RTI International, the University of Illinois, Chicago and the University of California, Los Angeles, USA.

Funding This work was supported by the following NIDA grants to the SATHCAP team: U01DA017387 to Yale University and the Biomedical Center and U01DA017377 to RAND Corporation.

Competing interests None.

Provenance and peer review Not commissioned; externally peer reviewed.

\section{REFERENCES}

1 AIDS Foundation East-West. HIV Statistics for Belarus, Moldova, Russia and Ukraine. 2008. http://www.afew.org/ knowledge-centre/hiv-statistics/ [accessed 18 July 2012].

2 UNAIDS (Joint United Nations Programme on HIV/AIDS)/ World Health Organization (WHO). Aids Epidemic Updatel Global Report. Switzerland, Geneva: UNAIDS/WHO, 2010.

3 Ministry of Health and Social Development of the Russian Federation. Country Progress Report of the Russian Federation on the Implementation of the Declaration of Commitment on HIV/AIDS. Russia: Moscow, 2008.

4 Zabina H, Kissin D, Pervysheva E, et al. Abandonment of infants by HIV-positive women in Russia and prevention measures. Reprod Health Matters 2009;17:162-170.

5 UNAIDS (Joint United Nations Programme on HIV/AIDS)/ World Health Organization (WHO). Aids Epidemic Update: December 2005. Special Section on HIV Prevention. Switzerland, Geneva: UNAIDS/WHO, 2005.

6 Remennick LI. Epidemiology and determinants of induced abortion in the U.S.S.R. Soc Sci Med 1991;33:841-848.

7 Ipas: Russia. Reproductive health and abortion statistics. In: International Data for Evaluation of Abortion Services. Chapel Hill, NC: Population Reference Bureau, 2007.

8 Regushevskaya E, Dubikaytis T, Nikula M, et al. Contraceptive use and abortion among women of reproductive age in St. Petersburg, Russia. Perspect Sex Reprod Health 2009;41:51-58.

9 Abdala N, Kershaw T, Krasnoselskikh TV, et al. Contraception use and unplanned pregnancies among injection drug-using women in St Petersburg, Russia. J Fam Plann Reprod Health Care 2011;37:158-164. 
10 Parfitt T. Russia moves to curb abortion rates. Lancet 2003;362:968.

11 Fedorova GV, Baniushevich IA. Factors impacting abortion prevalence. Probl Sotsialnoi Gig Zdravookhranenniiai Istor Med 2005;6:19-23.

12 Karelova GN. A reduction of abortions. Russian Federation. The Hague Forum. Integration 1999;60:29.

13 Sherwood-Fabre L, Goldberg H, Bodrova V. The impact of an integrated family planning program in Russia. Eval Rev 2002;26:190-212.

14 Perlman F, McKee M. Trends in family planning in Russia, 1994-2003. Perspect Sex Reprod Health 2009;41:40-50.

15 Zuckerman B. Developmental consequences of maternal drug use during pregnancy. NIDA Res Monogr 1985;59:96-106.

16 Balachova T, Bonner B, Chaffin M, et al. Women's alcohol consumption and risk for alcohol-exposed pregnancies in Russia. Addiction 2012;107:109-117.

17 Abdala N, Zhan W, Shaboltas AV, et al. Correlates of abortions and condom use among high risk women attending an STD clinic in St Petersburg, Russia. Reprod Health 2011;8:28.

18 Flanigan BJ, Hitch MA. Alcohol use, sexual intercourse, and an exploratory study. J Alcohol Drug Educ 1986;31:6-40.

19 World Health Organization (WHO). Global Status Report on Alcohol and Health. Geneva, Switzerland: WHO Press, 2011.

20 Boyles AL, DeRoo LA, Lie RT, et al. Maternal alcohol consumption, alcohol metabolism genes, and the risk of oral clefts: a population-based case-control study in Norway, 19962001. Am J Epidemiol 2010;12:924-931.

21 O'Leary CM, Nassar N, Kurinczuk JJ, et al. Prenatal alcohol exposure and risk of birth defects. Pediatrics 2010;126:e843e850.

22 Miller LC, Chan W, Litvinova A, et al. Fetal alcohol spectrum disorders in children residing in Russian orphanages: a phenotypic survey. Alcohol Clin Exp Res 2006;30:531-538.

23 Standerwick K, Davies C, Tucker L, et al. Binge drinking, sexual behaviour and sexually transmitted infection in the UK. Int J STD AIDS 2007;18:810-813.

24 Aicken CR, Nardone A, Mercer CH. Alcohol misuse, sexual risk behaviour and adverse sexual health outcomes: evidence from Britain's national probability sexual behaviour surveys. J Public Health (Oxf) 2011;33:262-271.

25 Connell CM, Gilreath TD, Hansen NB. A multiprocess latent class analysis of the co-occurrence of substance use and sexual risk behavior among adolescents. J Stud Alcohol Drugs 2009;70:943-951.

26 Ullman SE, Townsend SM, Starzynski LL, et al. Correlates of comorbid PTSD and polysubstance use in sexual assault victims. Violence Vict 2006;21:725-743.

27 Klanecky AK, Salvi S, McChargue DE. Coerced childhood sexual abuse moderates the association between cigarette smoking initiation and college drug use frequency. Am J Addict 2009; 18:363-366.

28 Shin SH, Edwards EM, Heeren T. Child abuse and neglect: relations to adolescent binge drinking in the national longitudinal study of Adolescent Health (AddHealth) Study. Addict Behav 2009;34:277-280.

29 Scott-Sheldon LA, Carey MP, Vanable PA, et al. Alcohol consumption, drug use, and condom use among STD clinic patients. J Stud Alcohol Drugs 2009;70:762-770.

30 Simbayi LC, Kalichman SC, Jooste S, et al. Alcohol use and sexual risks for HIV infection among men and women receiving sexually transmitted infection clinic services in Cape Town, South Africa. J Stud Alcohol 2004;65:434-442.

31 Booth RE, Lehman WE, Kwiatkowski CF, et al. Stimulant injectors in Ukraine: the next wave of the epidemic? AIDS Behav 2008;12:652-661.

32 Iguchi MY, Ober AJ, Berry SH, et al. Simultaneous recruitment of drug users and men who have sex with men in the United States and Russia using respondent-driven sampling: sampling methods and implications. J Urban Health 2009;86(Suppl. 1):5-31.

33 Magnani R, Sabin K, Saidel T, et al. Review of sampling hard-to-reach and hidden populations for HIV surveillance. AIDS 2005;19(Suppl. 2):S67-S72.

34 Abdul-Quader AS, Heckathorn DD, McKnight C, et al. Effectiveness of respondent-driven sampling for recruiting drug users in New York City: findings from a pilot study. J Urban Health 2006;83:459-476.

35 McKnight C, Des Jarlais D, Bramson $\mathrm{H}$, et al. Respondent-driven sampling in a study of drug users in New York City: notes from the field. J Urban Health 2006;83: i54-i59.

36 Bradley KA, DeBenedetti AF, Volk RJ, et al. AUDIT-C as a brief screen for alcohol misuse in primary care. Alcohol Clin Exp Res 2007;31:1208-1217.

37 Frank D, DeBenedetti AF, Volk RJ, et al. Effectiveness of the AUDIT-C as a screening test for alcohol misuse in three race/ ethnic groups. J Gen Intern Med 2008;23:781-787.

38 Raj A, Cheng DM, Krupitsky EM, et al. Binge drinking and unsafe sex: a study of narcology hospital patients from St. Petersburg, Russia. Subst Abus 2009;30:213-222.

39 Lavikainen HM, Lintonen T, Kosunen E. Sexual behavior and drinking style among teenagers: a population-based study in Finland. Health Promot Int 2009;24:108-119.

40 Cooper ML, Peirce RS, Huselid RF. Substance use and sexual risk taking among black adolescents and white adolescents. Health Psychol 1994;13:251-262.

41 David PH, Reichenbach L, Savelieva I, et al. Women's reproductive health needs in Russia: what can we learn from an intervention to improve post-abortion care? Health Policy Plan 2007;22:83-94.

42 Hillis SD, Rakhmanova A, Vinogradova E, et al. Rapid HIV testing, pregnancy, antiretroviral prophylaxis and infant abandonment in St Petersburg. Int J STD AIDS 2007;18:120-122.

43 Wolfe D, Carrieri MP, Shepard D. Treatment and care for injecting drug users with HIV infection: a review of barriers and ways forward. Lancet 2010;376:355-366.

44 Strandberg-Larsen K, Gronboek M, Andersen AM, et al. Alcohol drinking pattern during pregnancy and risk of infant mortality. Epidemiology 2009;20:884-891.

45 Kost K, Singh S, Vaughan B, et al. Estimates of contraceptive failure from the 2002 national survey of family growth. Contraception 2008;77:10-21.

46 Jones EF, Forrest JD. Contraceptive failure rates based on the 1988 NSFG. Fam Plann Perspect 1992;24:12-19.

47 Howard DE, Wang MQ, Yan F. Prevalence and psychosocial correlates of forced sexual intercourse among US high school adolescents. Adolescence 2007;42:629-643.

48 Zhan W, Hansen NB, Shaboltas AV, et al. Partner violence perpetration and victimization and HIV risk behaviors in St. Petersburg, Russia. J Trauma Stress 2012;25:1-8. 